\title{
Tangence
}

\section{Traces du Grand Meaulnes dans Le pont traversé (1921) de Jean Paulhan \\ Traces of Le Grand Meaulnes in Jean Paulhan's Le pont traversé (1921)}

\section{Camille Koskas}

Numéro 120, 2019

Le devenir-souvenir du roman. Poétique de la lecture romanesque

URI : https://id.erudit.org/iderudit/1069143ar

DOI : https://doi.org/10.7202/1069143ar

Aller au sommaire du numéro

Éditeur(s)

Tangence

ISSN

1189-4563 (imprimé)

1710-0305 (numérique)

Découvrir la revue

Citer cet article

Koskas, C. (2019). Traces du Grand Meaulnes dans Le pont traversé (1921) de Jean Paulhan. Tangence, (120), 79-93. https://doi.org/10.7202/1069143ar
Résumé de l'article

La présence du Grand Meaulnes dans le parcours de Jean Paulhan tient d'abord à un ensemble de faits biographiques : sa relation avec Jacques Rivière, beau-frère d'Alain-Fournier, après la guerre ; la profonde amitié qui le lie à Albert Uriet, dont il fait la rencontre en 1914, et qui sera le premier illustrateur du Grand Meaulnes. La correspondance qu'il échange avec ce dernier pendant la guerre témoigne de la place qu'occupe dans leur amitié la mémoire commune du roman d'Alain-Fournier, mémoire qui va irriguer et nourrir les écrits de cette période. Ces réminiscences du récit d’Alain- Fournier semblent se déposer dans un texte de Jean Paulhan paru en 1921, le Pont traversé, dans lequel l'auteur, par le dispositif qu'il met en place - des récits de rêves doublés d'un commentaire réalisé à l'état de veille par le narrateur, qui cherche à la fois à les extraire de l'oubli et à les élucider -, engage un processus de reconnaissance, d'exploration d'un espace intérieur, à la fois familier et étrange. Notre hypothèse est que ce processus de reconnaissance engagé par le récit puise à la fois dans cette mémoire partagée de la guerre avec Albert Uriet, qui s'inscrit dans leur correspondance, mais aussi dans les souvenirs du Grand Meaulnes, dont les traces mémorielles imprègnent profondément l'univers du Pont traversé. 
Tangence $\square \mathrm{n}^{\circ}$ 120, 2019 p. 79-93.

\author{
Traces du Grand Meaulnes \\ dans Le pont traversé (1921) de Jean Paulhan \\ Camille Koskas \\ Sorbonne Université
}

Je souhaite m'intéresser ici aux traces du Grand Meaulnes ${ }^{1}$ dans un récit de Jean Paulhan écrit pendant la guerre alors qu'il était mobilisé au front, Le pont traversé2 ${ }^{2}$ publié en $1921^{3}$. Les rares études qui se sont attaquées au commentaire des récits de Paulhan ont souligné leur caractère hermétique, déstabilisant: difficultés qui tiennent, comme le souligne Michel Murat ${ }^{4}$, à la fois à l'embarras que l'on trouve à les inscrire dans une histoire littéraire et à les rattacher à une famille de textes, à la fois à leur ambiguïté générique (ni roman, ni nouvelle, ni pure fiction, ni autobiographie), mais aussi au caractère bien souvent énigmatique de leur signification. Obscurité toute

1. Je me réfère à l'édition suivante: Alain-Fournier, Le Grand Meaulnes, présentation de Tiphaine Samoyault, Paris, Garnier-Flammarion, 2009. Désormais, les références à cet ouvrage seront indiquées par le sigle GM, suivi de la page, et placées entre parenthèses dans le corps du texte.

2. Jean Paulhan, Le pont traversé, dans Euvres complètes, éd. Bernard Baillaud, Paris, Gallimard, coll. «Blanche», t. I (Récits), 2006, p. 145-157. Désormais, les références à cet ouvrage seront indiquées par le sigle $P T$, suivi de la page, et placées entre parenthèses dans le corps du texte.

3. Cette réflexion m'a été suggérée par Claire Paulhan, avec qui je travaille à l'Institut Mémoire de l'édition contemporaine (IMEC) sur un projet de numérisation des correspondances de Jean Paulhan, projet que je mène avec Clarisse Barthélemy dans le cadre des travaux du Labex-Observatoire de la vie littéraire (OBVIL) de Sorbonne Université.

4. Michel Murat, "Progrès dans le roman assez lent», dans Clarisse Barthélemy (dir.), La littérature selon Jean Paulhan, Paris, Classiques Garnier, 2014, p. 135-151. 
sémantique, qui renvoie au questionnement soulevé par le sens de la narration, puisque Paulhan utilise une syntaxe absolument claire, pour dépeindre des éléments parfaitement nets. Parmi ces récits, $L e$ pont traversé est pour Jean-Yves Tadié l'un des plus difficiles ${ }^{5}$, qu'il range au côté de Mélusine de Hellens ${ }^{6}$, ou Vagadu ${ }^{7}$ de Jouve, parmi ces textes constitués de "systèmes de rêves" " .

L'importance accordée au rêve dans les récits de Paulhan n'est guère isolée. Dans Le guerrier appliqué et Lalie, deux récits écrits pendant les années de guerre, le rêve occupe une place considérable, en ce qu'il y mord sur la réalité et l'éveil - le narrateur du Guerrier appliqué s'y réveille encore mal dégagé de rêves, et les scènes de rêve et d'éveil dans Lalie mettent en évidence la frontière poreuse entre les deux états. Jean-Yves Tadié prend aussi l'exemple des Causes célèbres, dont la section intitulée "Un réveil dans le rêve», où le héros ne sait s'il a échappé au rêve, puisqu'il a rêvé qu'il s'éveillait, permet de rassembler deux états ou principes contradictoires selon un dispositif cher à Paulhan: Tadié décrit le récit de Paulhan comme une "expérience contradictoire» dont le sens se situe «dans le choc de deux éléments qui s'annulent en produisant l'étincelle poétique ${ }^{9} »$. Le statut du rêve dans Le pont traversé est cependant différent, puisque ces deux éléments, comme le souligne Jean-Yves Tadié, y sont organisés en système. Divisé en trois sections qui correspondent à trois nuits, le récit est structuré par deux mouvements: l'un est un mouvement de va-et-vient entre le rêve et son commentaire éveillé, lisible dans la structure typographique du texte (italique pour la phase d'éveil, romain pour le rêve); l'autre, un mouvement de progression, d'avancée, comme le titre le laisse attendre, le texte se lisant alors comme un trajet initiatique dont le narrateur enregistre les progrès. La troisième nuit se conclut ainsi sur ces mots: «[I]l me paraissait

5. Jean-Yves Tadié, Le récit poétique, Paris, Presses universitaires de France, coll. «Écriture», 1978, p. 176.

6. L'édition définitive de ce roman de Franz Hellens est parue en 1952, avec le sous-titre «Roman d'aventure», que l'auteur présente comme le produit d'une «somme de rêves». Vingt-six chapitres constituent une juxtaposition d'aventures oniriques.

7. Ce récit, paru en 1931, se présente comme une succession de scènes oniriques rêvées par Catherine Crachat, également héroïne d'Hécate (1928), alors qu'elle est en psychanalyse.

8. Jean-Yves Tadié, Le récit poétique, ouvr. cité, p. 172; l'expression «système de rêve» est empruntée à Pierre Jean Jouve dans En miroir (1954).

9. Jean-Yves Tadié, Le récit poétique, ouvr. cité, p. 137. 
maintenant que j'avais gagné, au cours des premiers rêves, une telle science, et par elle l'avantage sur quelque rival» ( $P T$, p. 156). Ce deuxième mouvement fait du Pont traversé un récit aimanté, tendu vers les retrouvailles avec l'être aimé, à qui est adressé le texte et dont l'éloignement a mis en marche le récit. Les neuf rêves dont le narrateur fait le récit sont donc autant d'étapes d'une quête amoureuse, tentative pour renouer avec une figure aimée que la "gêne», les «reproches», la «rancune» ont éloignée.

Cette logique de la quête - pour déconcertante et atypique qu'elle soit chez Paulhan, en raison de la brièveté de la forme, du caractère énigmatique de l'objet recherché, mais aussi de la part prise par le langage dans cette quête — inscrit Le pont traversé parmi ces romans de l'aventure intérieure que Jacques Rivière définit dans la Nouvelle revue française en $1913^{10}$, et dont Le Grand Meaulnes d'Alain-Fournier constitue bien sûr un des archétypes. Par le dispositif mis en place, qui double les récits de rêves d'un commentaire et cherche à la fois à les extraire de l'oubli et à les élucider, le texte engage un processus de reconnaissance, d'exploration d'un espace intérieur, à la fois familier et étrange. L'hypothèse que je souhaiterais développer ici est que ce processus de reconnaissance puise dans les souvenirs du Grand Meaulnes, dont je chercherai à prouver que les traces imprègnent profondément l'univers du Pont traversé. Mais j'aimerais montrer que ces réminiscences passent elles-mêmes par la médiation d'une autre expérience: il s'agit de l'expérience de la guerre, et de l'amitié que Paulhan noue avec Albert Uriet, qui s'établit sur la constitution d'une mémoire commune autour du roman d'Alain-Fournier, mémoire qui va irriguer et nourrir les récits écrits pendant la guerre.

\section{La guerre et la rencontre avec Albert Uriet}

Le 26 août 1915, Uriet écrit à Paulhan, alors que les deux amis ont été séparés pour être mobilisés à des endroits différents: «Jamais je n'ai tant éprouvé ce que sentait François Seurel après le départ

10. Sur ce point, voir en particulier Maaike Koffeman, Entre classicisme et modernité. La Nouvelle revue française dans le champ littéraire de la Belle Époque, Amsterdam/New-York, Rodopi, 2003; et Christophe Pradeau, «L'état d'aventure», Études littéraires, vol. 44, $\mathrm{n}^{\circ} 1$ (L'aventure comme possibilité. Le roman français de la première moitié $d u x^{e}$ siècle, dir. Mathieu Bélisle), hiver 2013, p. 55-66. 
d'Augustin Meaulnes. Et je vois avec un grand plaisir Saudon, parce qu'il était de "ton temps". Adieu donc, mon grand Meaulnes, écrismoi vite, je t'en prie.» Et il ajoute: «Ce matin, en m'éveillant, un rayon transformait mon vieux gilet en habit d'or; de savoir le vide de ce jour, j'ai eu un serrement de cœur ${ }^{11}$ ", évocation qui me semble une référence explicite au gilet de soie du Grand Meaulnes, seule relique qu'il conserve de la fête merveilleuse lors de son retour au village. Cette double référence au Grand Meaulnes, qui condense deux passages différents du texte, n'est guère isolée sous la plume d'Albert Uriet. Comme on le voit, elle est chargée d'une dimension affective profonde: plus que simple souvenir de lecture, elle semble investie d'une nécessité presque vitale, et constituer une mémoire commune sur laquelle va s'établir l'amitié avec Paulhan.

Lorsqu'Uriet écrit cette lettre à Paulhan en 1915, ils viennent de se rencontrer, quelques mois plus tôt, au fort de Beauvais, où ils sont tous les deux mobilisés. Né en 1889, Uriet est un dessinateur, passé par l'École des Arts décoratifs de Paris. L'amitié très forte qu'il noue avec Paulhan en 1915 se double d'une complicité amoureuse, puisque l'un et l'autre sont épris d'une certaine Germaine. L'étude de leur correspondance, déposée à l'Institut Mémoire de l'édition contemporaine (IMEC), montre d'ailleurs que cette fusion amicale se traduit par un mimétisme dans la graphie des épistoliers. Cette amitié va jouer aussi un rôle essentiel dans l'écriture des récits paulhaniens. Uriet transmet en effet à Paulhan certains souvenirs d'enfance, les observations recueillies lors de ses promenades, que ce dernier réinvestira dans Lalie ou dans Le guerrier appliqué ${ }^{12}$. Ces deux textes, mais aussi Le pont traversé seront illustrés par Uriet, même si ces versions ne donneront pas lieu à des publications, sauf pour Le guerrier appliqué13. Les deux amis se livrent également à l'écriture d'un roman commun dont la parution ne verra jamais le jour.

En 1919, Paulhan transmet à Rivière, beau-frère d'AlainFournier, une idée d'Albert Uriet pour illustrer le Miracle des trois dames de village. Les illustrations d'Uriet sont caractérisées par

11. Lettre d'Albert Uriet à Jean Paulhan du 26 août 1915, inédit, IMEC.

12. Voir Bernard Baillaud, «Notice» de Lalie et «Notice» du Guerrier appliqué, dans Jean Paulhan, Euvres complètes, ouvr. cité, p. 453-461 et p. 489-497. Baillaud cite par exemple une lettre d'Uriet à Paulhan envoyée en septembre 1915 (p. 495): "Montgomery nous a raconté des histoires durant tout le repas. Il y aurait là des épisodes pour ton "guerrier appliqué”.»

13. Dans le cas du Pont traversé et de Lalie, la version illustrée n’a jamais été publiée. 
leurs couleurs chatoyantes, leur inspiration rurale (motifs de villages, de collines, de moulins) et les personnages de fantaisie qu'elles convoquent (gnomes, lutins). Ses illustrations se nourrissent aussi bien souvent de ses lectures d'enfance, comme Robinson Crusoé, qu'il illustre en 1923, et qu'il évoque à plusieurs reprises dans sa correspondance avec Paulhan. Par exemple, le 27 septembre 1915, alors qu'il est de garde toute la nuit, il écrit: «Le vent hurlait dans la plaine et faisait craquer les arbres (je pensais à Robinson Crusoé, aux pages où il a la fièvre et où il boit une décoction de tabac en guise de remède $[\ldots])^{14}$.» La figure de Robinson est à nouveau évoquée dans une lettre du 18 mai 1925:

Il y a, près de mon lit, une espèce d'alcôve [...]. C'est le dessous d'un escalier de bois. Quand quelqu'un y passe, cela fait un bruit sonore, on dirait les coups qu'on frappe au théâtre. Je me dis: «La Rancune, sortez de dessous votre rideau et venez tourner autour de ma chandelle, ou Robinson Crusoé, ou Capitaine Anson.» Ils viennent tous, sans laisser d'ombre sur le mur ${ }^{15}$.

Rappelons l'importance de Robinson Crusoé dans Le Grand Meaulnes, personnage auquel le narrateur compare Meaulnes perdu dans ses réflexions, au tout début du roman. Meaulnes comparé à Crusoé par Alain-Fournier, Paulhan comparé à Meaulnes par Uriet: on voit se déployer une sorte de chaîne de l'identification, du jeu de rôle, qui repose sur la constitution d'une bibliothèque mentale.

En 1925, Uriet travaille aux illustrations du Grand Meaulnes, qui donnera lieu à la première édition illustrée du roman pour le compte de l'Association des médecins bibliophiles. La suite de son parcours, où l'amitié avec Paulhan se dénoue, nous intéresse moins dans le cadre de cet article. On évoquera simplement ses activités de directeur artistique de la maison d'édition catholique Mame, qu'il prend en charge à partir de 1930, et où il œuvre en particulier à promouvoir les contes, en contribuant aux principaux recueils illustrés qui paraissent. Il bataille également pour faire reconnaître le talent d'Henri Pourrat dont il est très proche, et dont il illustre deux contes qui paraissent en 1936. La dernière partie de son existence sera assez solitaire: Uriet se désengage progressivement de ses responsabilités artistiques et éditoriales et meurt en 1954.

14. Lettre d'Uriet à Paulhan du 27 septembre 1915, inédit, IMEC.

15. Lettre d'Uriet à Paulhan du 18 mai 1925, inédit, IMEC. 
Qu'en est-il maintenant de la présence du Grand Meaulnes dans l'itinéraire de Jean Paulhan? À notre connaissance, il n'a consacré aucun texte critique à ce roman. De manière plus étonnante, il n'est pas non plus question du Grand Meaulnes dans la correspondance que Paulhan échange avec Jacques Rivière, qu'il a rencontré après la guerre, sauf quand il lui demande, en 1920, de mettre au net les manuscrits laissés par son beau-frère, qui seront publiés sous une forme inachevée en décembre 1922 sous le titre Colombe Blanchet. La présence du Grand Meaulnes dans l'itinéraire de Paulhan tient donc avant tout à un ensemble de faits biographiques, mais aussi de traces, d'échos diffus: l'amitié avec Rivière et Uriet, la librairie achetée par sa première femme, Sala, située sur le boulevard du Montparnasse à Paris, et baptisée Au Grand Meaulnes, l'Association des amis d'Alain-Fournier à laquelle Paulhan participe pendant la guerre et qui sert de cache à ses activités de résistance. La présence du Grand Meaulnes semble donc infuser discrètement au sein de l'itinéraire de Paulhan, se déposer dans certains signes, certains nœuds, sans pour autant faire l'objet d'un engouement explicite, ni même être évoquée sous la forme de simples souvenirs de lecture, mis à part dans la correspondance avec Uriet, où le souvenir de Meaulnes est convoqué explicitement à plusieurs reprises. C'est donc à travers cette amitié et cette correspondance que s'élabore pendant la guerre une mémoire commune, qui alimentera la création littéraire, où Uriet, rappelons-le, sera triplement impliqué, en tant qu'illustrateur, co-auteur et source d'inspiration. Et c'est par le truchement de cette mémoire commune que l'univers du Grand Meaulnes déteindra sur les récits paulhaniens et les contaminera.

\section{Le Grand Meaulnes dans la correspondance Uriet-Paulhan}

Comme nous l'avons vu, la présence du Grand Meaulnes est explicitement convoquée dans la correspondance par Uriet, désignée à la fois comme un des moteurs de la création, qu'elle soit artistique ou romanesque (Uriet évoque ainsi dans une lettre à Paulhan la recherche difficile d'un «charme indéfinissable», à laquelle le souvenir du Grand Meaulnes empêchera de renoncer ${ }^{16}$ ), et comme une présence parfois trop envahissante, en ce que son souvenir écrasant empêche d'apprécier d'autres œuvres: «[J]'ai relu quelques passages

16. Lettre d’Uriet à Paulhan du 28 septembre [année non précisée], inédit, IMEC. 
de la légende de Gösta Berling ${ }^{17}$. Je l'aime beaucoup plus que la première fois. Mais j'étais encore trop plein du "Grand Meaulnes" pour apprécier cette vie des cavaliers ${ }^{18}$.» L'univers du Grand Meaulnes est aussi évoqué à travers une série de comparaisons, qui renvoient à des scènes clés du livre: départ de Meaulnes et sentiment de désarroi et de solitude de François Seurel, dans lequel, nous l'avons vu, se reconnaît Uriet après sa séparation d'avec Paulhan; domaine de Noirat que visite Uriet, où se trouve « une vieille maison abandonnée tout à fait étrange», qui lui rappelle un peu celle du Grand Meaulnes: «Des livres dorés, des vieux chapeaux, deux anciennes vestes de papetier bleue et verte, avec des boutons de métal, une vieille horloge à pois, des dressoirs, des bahuts - et une cheminée étonnante avec un banc, une crémaillère, et un poële à frire dont la queue dépasse $1 \mathrm{~m}$ de long ${ }^{19}$.» Ce passage peut évoquer l'arrivée de Meaulnes au domaine mystérieux, où il découvre dans une chambre un grand lit bas, couvert de vieux livres dorés.

On soulignera que cette évocation s'accorde par ailleurs avec les observations faites par Alain Buisine dans son ouvrage Les mauvaises pensées $d u$ Grand Meaulnes ${ }^{20}$, lorsqu'il relève que c'est toujours la première partie du livre d'Alain-Fournier qui est louée et célébrée au détriment de tout le reste de l'œuvre, comme si le roman programmait lui-même l'oubli du réel au profit de la féerie. Alain Buisine souligne que Gide en faisait également la remarque dans son journal en date du 2 janvier 1933. Il y jugeait en effet le « dessin du texte quelque peu incertain et dont le plus exquis s'épuise dans les cent premières pages», sans que la suite du récit parvienne à ressaisir cette fraîcheur des débuts ${ }^{21}$. "Il se trouve que c'est toujours la seconde [partie] qu'on oublie. Que c'est la partie humaine qui s'efface au profit de la construction fantastique qui, par avance, a réduit ses pouvoirs et annihilé son développement ${ }^{22}$.» Rappelons que, pour

17. Roman suédois de Selma Lagerlöf paru en 1891. Il s'agit du récit des aventures d'un pasteur qui se mêle à une bande d'aventuriers après avoir été chassé de son presbytère pour ivrognerie.

18. Lettre d'Uriet à Paulhan du 2 mars 1916, inédit, IMEC.

19. Lettre d'Uriet à Paulhan, non datée, inédit, IMEC.

20. Alain Buisine, Les mauvaises pensées du Grand Meaulnes, Paris, Presses universitaires de France, coll. «Le texte rêve», 1992.

21. Journal d'André Gide cité par Alain Buisine, Les mauvaises pensées du Grand Meaulnes, ouvr. cité, p. 18.

22. Alain Buisine, Les mauvaises pensées du Grand Meaulnes, ouvr. cité, p. 20. 
Buisine, ce trajet mémoriel dépend moins de la sensibilité de chaque lecteur que d'un dispositif d'idéalisation à l'œuvre dans le texte, «machine à faire oublier sa seconde partie ${ }^{23}$ ", qui s'emploierait à graver dans l'esprit du lecteur la féérie de la fête étrange au détriment des trivialités du réel, dans un processus de sublimation et de refoulement de ce que le texte contient d'impur. Mais je ne m'étendrai pas sur l'hypothèse défendue par Alain Buisine, cette parenthèse visant simplement à montrer que la question de la mémoire du roman a été au centre des analyses et des commentaires qui ont été dédiés au Grand Meaulnes.

Les réminiscences de Meaulnes exposées dans la correspondance ne font d'ailleurs pas exception à cette «règle» observée par Gide puis par Buisine: c'est bien l'espace de la féerie, du merveilleux qui est investi comme espace consolateur et appelé en renfort pour adoucir l'ennui et l'horreur de la guerre. Parallèlement aux références explicites à l'univers et aux personnages du Grand Meaulnes, l'espace entier de la correspondance est captivé par ces réminiscences, qui semblent inspirer les contes aux accents merveilleux inventés par Uriet. Une attention extrême est portée à la nature, au rythme des saisons, à la peinture de paysages de «brumes dorées» balayées par de longs flots de vent. Au sein des descriptions du quotidien du soldat, souvent très minutieuses (description de la nourriture, indication des prix du lit), sont ménagés des seuils, des échappées sous forme de peintures de la nature, souvent contaminées par le merveilleux. Le souvenir du Grand Meaulnes semble jouer le rôle d'un filtre qui oriente le regard, façonne la perception, adoucit aussi, peut-être, les réalités brutales de la guerre.

Le 5 février 1915, Uriet reprend ainsi les images maritimes propres à l'univers du Grand Meaulnes, notant que «[1] a grange est comme un beau navire: hier, il fendait tranquille et sûr, de longs flots de vent ${ }^{24} »$. Il s'abandonne ensuite à une description des toits ( «la tour simple et massive d'une église, entourée de toits gris et rouges, en deçà des prairies, quelques longs arbres, et une ligne de saules aux branches déjà chaudes et brunes»), avant de revenir à la réalité de la guerre: «Et toujours des aéroplanes allemands

23. Alain Buisine, Les mauvaises pensées du Grand Meaulnes, ouvr. cité, p. 15.

24. Lettre d'Uriet à Paulhan du 5 février 1915, inédit, IMEC; dans Le Grand Meaulnes, c'est la salle de classe qui est comparée, au tout début du chapitre IV ( "L'évasion»), à une barque sur l'Océan, sillonnant un paysage gelé. 
passent, accompagnés d'obus et de balles.» Sont dépeints des paysages de maisons abandonnées et de chemins déserts, l'image de la route, du sentier, constituant de manière récurrente le support de micro-récits. Sur ce point, rappelons l'omniprésence, dans Le Grand Meaulnes, de la dimension topographique, et l'importance des chemins, sentiers, qui découpent le paysage, sans pour autant le rendre plus rationnel (un des chapitres du Grand Meaulnes s'intitule d'ailleurs «À la recherche du sentier perdu»). Le chemin, dans la correspondance, constitue l'un de ces seuils où se joue le basculement vers l'espace de la féérie et du conte. Le 7 décembre 1916, Uriet écrit ainsi :

On a pris le chemin qui passe sous le chemin de fer; et tout de suite ce vent pointu du Nord sur le côteau glacé. Frappe contre l'arbre, vieux petit homme, puis regarde-nous. Mais quelle étonnante culotte blanche et noire! Nous avons continué notre route (lui, empoignant l'arbre par les cheveux, tira si fort, si fort, que l'arbre tomba avec une sorte de gémissement ${ }^{25}$.

Il évoque ensuite le chemin bordé de masures et de vieux noyers qu'il parcourt et qui mène à une masure abandonnée, où Uriet espère retrouver Paulhan et Germaine; son récit mobilise alors, semble-t-il, le souvenir du Grand Meaulnes arpentant les sentiers de Sologne au milieu de la campagne glacée et sa rencontre avec un couple de vieux paysans, première étape du basculement de son parcours dans la féérie jusqu'à l'arrivée au domaine mystérieux.

Chez Uriet, la route fonctionne à la fois comme une échappée vers l'imaginaire et un puissant vecteur de rencontre. Le 19 décembre 1915, croisant une jeune femme qui se retourne pour lui envoyer un baiser, il se rêve un instant Meaulnes, rencontrant sur le chemin qui longe le domaine Yvonne de Galais escortée d'une vieille femme bossue:

Sur cette route, où, décidément, il ne passe que quelques vieilles femmes et qui disparait tout de suite derrière des buissons je me suis croisé avec une jeune fille. Cela m’a semblé si extraordinaire que je lui ai adressé le bonjour. Nous avons causé un instant. Elle allait au village de Câtillon. Je voudrais la revoir. Mais j'ai le sentiment qu'elle ne passera là jamais plus ${ }^{26}$.

25. Lettre d'Uriet à Paulhan du 7 décembre 1916, inédit, IMEC.

26. Lettre d'Uriet à Paulhan du 19 décembre 1915, inédit, IMEC. 
Ces figures de jeunes femmes évanescentes lui inspirent même l'idée d'un roman en 1915, qui rappelle la figure affaiblie d'Yvonne de Galais à la fin du roman: "Je voudrais mettre un charme très prenant autour d'une jeune fille maladive - connue jadis.» Mettant en scène trois enfants, découvrant ensemble ce qu'il nomme «le mystère de leur sexe», Uriet croque également une scène, qui serait pour Buisine le grand interdit du Grand Meaulnes: «J'ai assisté, ce matin, au bout de mes jumelles, à une petite scène amusante (une petite fille et deux petits garçons se découvrant le mystère de leur sexe, sous un pommier — cela avait un petit air païen — je t'assure que c'était délicieux $[\ldots])^{27}$.» Irruption de la sexualité dans le monde de l'enfance, cette scène pourrait se lire comme l'envers heureux, naïf, inoffensif du trio amoureux constitué par Franz-Meaulnes-Valentine, réunis par une sexualité maudite en ce qu'elle signe l'irrémédiable fin de l'enfance. Les figures enfantines tiennent d'ailleurs une place de choix dans les lettres d'Uriet comme dans celles de Paulhan, et constituent un des points de bascule, un des lieux de passage du réel à la fiction puisqu'elles nourrissent et peuplent les contes qu'ils écrivent pendant la guerre - que l'on songe à Lalie, petite fille réelle dont Paulhan a fait un récit.

\section{De la guerre au Pont traversé}

Notre hypothèse est donc que ces réminiscences du texte d'Alain-Fournier, collectées et entretenues pendant la guerre, se déposeraient dans Le pont traversé, écrit pendant la guerre. À l'instar du Grand Meaulnes, le récit est structuré comme une quête amoureuse. L'éloignement de l'être aimé qu'il faut retrouver s'explique à la fois par un défaut du langage et par le sentiment plus obscur d'une faute commise par le narrateur, qui évoque les reproches dont il est la cible. La figure des rivaux, «masculins» et «féminins», constitue un obstacle récurrent. L'un et l'autre apparaissent lors de la troisième nuit: rival masculin, sur qui le narrateur prend l'avantage, et mise en scène de la rivalité féminine sous la forme de deux jeunes femmes à cheval dans le dernier rêve, qui convoque, peut-être, le souvenir d'Yvonne de Galais montée en amazone sur le vieux Bélisaire, lors de ses décevantes retrouvailles avec Meaulnes. L'interrogation lancée par le narrateur au début de cette section — «J'aimais peut-être

27. Lettre d'Uriet à Paulhan du 10 septembre 1915, inédit, IMEC. 
cette jeune femme; ou bien était-ce une amie, ou ma sœur?» (PT, p. 156) - peut évoquer le statut trouble des figures féminines dans Le Grand Meaulnes, Yvonne de Galais incarnant à la fois l'amie, la sœur et l'amoureuse, à laquelle vient se superposer la figure d'Isabelle Rivière, à qui le livre est dédié, sœur d'Alain-Fournier et épouse de son ami intime Jacques Rivière.

Le récit de rêve est l'occasion de déployer des paysages de campagnes gelées et de villages abandonnés qui paraissent puiser à cette mémoire, qui s'est constituée et solidifiée pendant la guerre autour du souvenir du Grand Meaulnes: présence de la nature et attention aux saisons; paysages décrits en surplomb, rappelant les descriptions faites par Uriet les soirs de guet (les toits); présence insistante du village inconnu, village obscur qui titre l'une des sections du texte de Paulhan et qui peut rappeler le chapitre du Grand Meaulnes intitulé «Le domaine mystérieux» (chapitre xI); animation merveilleuse de l'inanimé, par exemple le fagot de bois qui tient le cou d'une bûcheronne comme un enfant ( $P T$, p. 154), et qui rappelle l'arbre qu'on tire par les cheveux et qui tombe avec un gémissement évoqué par Uriet dans ses lettres. La section «La promenade rapide» en direction du village inconnu, racontée lors de la deuxième nuit du Pont traversé, semble faire écho au trajet en bicyclette entrepris par le narrateur du Grand Meaulnes à la recherche du domaine mystérieux : «Tout prend part à sa vitesse; le toit de l'église fait suite aux toits des maisons; ils ont poussé en même temps, ils partent de deux vieilles courbées et bavardes contre un puits.» (PT, p. 153) Dans Le Grand Meaulnes, la promenade à bicyclette est décrite ainsi: «Du haut des côtes, descendre et s'enfoncer dans le creux des paysages; découvrir comme à coups d'ailes les lointains de la route qui s'écartent et fleurissent à votre approche, traverser un village dans l'espace d'un instant et l'emporter tout entier d'un coup d'œil.» (GM, p. 157) D'ailleurs, le narrateur du Grand Meaulnes ne note-t-il pas: «En rêve seulement j'avais connu jusque-là course aussi charmante, aussi légère»? (GM, p. 157)

Se tisse aussi, au sein du texte, toute une topographie intérieure qui investit particulièrement tous ces lieux de passage entre le dedans et le dehors, le familier et l'étranger, que sont chemins, sentiers et ponts, explicitement présentés par Paulhan comme des métaphores de l'existence, à l'instar des «sentiers perdus» sur lesquels cheminent les personnages du Grand Meaulnes. Cette extrême 
mobilité qui caractérise le récit paulhanien donne lieu à la rencontre avec toute une population, qui rappelle les figures croisées par le Grand Meaulnes, mais aussi les notes prises par Uriet pendant la guerre. La figure de la jeune fille - d'ailleurs devenue jeune femme à la fin du texte - ouvre et ferme le récit de Paulhan. Entre-temps, le narrateur croise régulièrement des personnages de vieil homme et de vieille femme rappelant les figures de vieux petits hommes et de sorcières convoquées par Uriet dans ses lettres. Que l'on songe par exemple à la bûcheronne au fagot de bois dans «La jeune fille dans la forêt» $(P T$, p. 154), qui rappelle le paysan âgé et sa brassée de bois dans Le Grand Meaulnes (GM, p. 49).

Reflet peut-être et prolongement de la séparation du narrateur d'avec l'être aimé, les figures masculines et féminines, également, sont souvent séparées, ce qui n'est pas d'ailleurs sans provoquer une certaine inquiétude: le narrateur remarque, au sujet de l'assemblée du peuple onirique des Nifis, qu' «il n'y a pas de femmes parmi eux, ce qui rend leur langage plus rude» (PT, p. 155), ou évoque «une réunion habituelle d'hommes, comme une popote», lors de la première nuit ( $P T, \mathrm{p} .147)$. Les figures féminines restent obstinément silencieuses (la jeune fille de la première section ne répond pas au narrateur quand il s'adresse à elle), et toutes les paroles proférées dans le récit sont masculines. Là où les hommes croisés par le narrateur entrent en contact avec lui par la parole, les femmes sont l'objet d'un rapport exclusivement visuel: il observe, du haut d'une terrasse, les jeunes femmes qui passent à cheval ( $P T$, p. 157) ou encore, dans la forêt, une jeune fille qui se confond avec le tapis des feuilles (PT, p. 155). La vision de celle-ci provoque d'ailleurs chez le narrateur une joie d'une intensité inattendue, rappelant le «contentement extraordinaire », inexplicable qui s'empare du Grand Meaulnes à l'approche du domaine mystérieux. C'est d'ailleurs à cette seule occasion que Paulhan emploie dans son récit le terme d' "aventure», omniprésent dans le texte d'Alain-Fournier et étroitement rattaché à l'article de Rivière. Le pont traversé pourrait donc s'inscrire dans ces romans de l'aventure intérieure tels que Rivière les a définis. Le narrateur y explore à tâtons le labyrinthe de son imagination, dans une quête qui s'inscrit dans une double logique: mouvement vers l'inconnu et reconnaissance du familier. C'est sur ce point, qui me paraît le plus remarquable dans la perspective du rapprochement avec Le Grand Meaulnes, que je souhaiterais à présent m'attarder. 
La question de la reconnaissance est en effet centrale dans le récit, et le terme y est régulièrement invoqué. Dans «Le village obscur", il est question d'un village que le narrateur n'a vu que la nuit, et qu'il n'est pas sûr de reconnaître en plein jour. Le rêve donne lieu, une fois éveillé, à une interrogation qui déplace la question de la reconnaissance vers la reconnaissance amoureuse: «Un jour peut-il venir où je ne te reconnaîtrai pas?» $(P T, \mathrm{p} .149)$ Dans la première section de la deuxième nuit, le narrateur indique que les hommes rencontrés par un vieillard "négligent» de le reconnaître (PT, p. 151). La deuxième section de cette même deuxième nuit s'intitule «La fausse reconnaissance»: un homme, croyant reconnaitre le narrateur, lui demande de manière insistante s'il vient de Bouillargues. La scène rappelle l'arrivée du Grand Meaulnes chez le couple de paysans qui l'accueillent avant son arrivée au domaine et devant qui il cherche à cacher qu'il n'est pas du pays, jusqu'à ce que la vieille femme lui demande: "C'est-il que vous n'êtes pas du pays?» (GM, p. 49) Sans même invoquer la psychanalyse, on sait qu'Alain-Fournier multiplie dans Le Grand Meaulnes les signes qui invitent à voir dans le domaine mystérieux le retour d'un territoire de l'enfance, envers lequel Meaulnes éprouve une familiarité trouble. C'est d'ailleurs bien souvent par le truchement du rêve que s'exprime cette familiarité: à son arrivée au domaine, il se rappelle ainsi d'un rêve d'enfant mettant en scène une jeune fille semblant attendre son réveil (GM, p. 53), et l'on sait combien le thème du réveil dans le rêve fascine Paulhan. Meaulnes, de même, perçoit la fête étrange comme un rêve lui rappelant son rêve de jadis (GM, p. 69). La topique de la reconnaissance amoureuse est mobilisée lors de la première rencontre entre Yvonne et Meaulnes, où le regard de la jeune femme semble signifier à ce dernier: "[J]e ne vous connais pas. Et pourtant il semble que je vous connais.» (GM, p. 73)

Comme nous l'avons déjà remarqué, la section «Le village obscur» est l'une de celle où la question de la reconnaissance - reconnaissance des lieux, reconnaissance de l'être aimé - est évoquée avec le plus d'insistance, s'accompagnant d'ailleurs d'un sentiment d'inquiétude: "Je n'étais jamais venu dans ce village que le soir. Ma familiarité avec lui se mélangeait de la crainte où je demeurais de ne pas le reconnaître, s'il m'arrivait de le voir en plein jour.» (PT, p. 148) On relève dans cette citation l'union de deux principes contraires, la familiarité et le sentiment de l'étrangeté, d'autant que, 
paradoxalement, c'est de jour, et non de nuit, que le narrateur craint de ne pas reconnaître le village. «Le village obscur» est aussi, me semble-t-il, la section qui puise le plus directement dans l'imaginaire du Grand Meaulnes, à commencer par le titre, qui rappelle ce chapitre clé du Grand Meaulnes intitulé «Le domaine mystérieux» (GM, p. 53). L'arrivée du narrateur dans ce village rappelle, comme nous l'avons signalé, la halte faite par Meaulnes chez un couple de paysans avant d'arriver au domaine; le chapitre du Grand Meaulnes intitulé «Une halte» (chapitre Ix, p. 47) se conclut d'ailleurs sur l'évocation d'une cour obscure. On retrouve dans le texte de Paulhan la bonne femme et le chien furieux du Grand Meaulnes, alors que Le pont traversé dépeint un chien qui garde hargneusement un tas de pommes, qui accompagne une vieille femme. Comme dans Le Grand Meaulnes, ce sont les lumières aux fenêtres des maisons qui permettent au narrateur de se guider dans l'obscurité. Comme Meaulnes enfin, au moindre écart, le narrateur risque de perdre de vue les maisons: «Je me trouvai revenir, pour cette seule fois un matin, vers le village. (Je ne m'en étais pas éloigné la nuit, ou très peu de sorte que je n'avais pas de doute.)» (PT, p. 148) Rappelons que, parti chercher sa jument pour l'amener à l'écurie, Meaulnes ne parvient pas à retrouver la trace de la maison où le couple de paysan l'a accueilli.

Cet ensemble de remarques n'autorise évidemment pas à voir dans Le pont traversé une relecture ou une réécriture du Grand Meaulnes. La question du langage, centrale dans le texte de Paulhan, l'éloigne des thématiques d'Alain-Fournier, et en fait quelque chose de tout à fait singulier et différent. Il faut aussi, bien sûr, prendre garde aux projections de notre propre mémoire de lecteur sur les textes. Il me semble en revanche que le récit puise à cette mémoire commune, qui s'est constituée et consolidée pendant la guerre avec Albert Uriet autour des souvenirs du texte d'Alain-Fournier. Le souvenir de lecture, associé à cet espace protecteur qu'est la chambre, $\mathrm{y}$ est d'ailleurs souvent convoqué, rempart contre les souffrances de la guerre. Uriet écrit ainsi dans une lettre du $1^{\text {er }}$ septembre 1915:

Au temps où j'habitais chez mes tantes, $\mathrm{j}$ 'adorais les soirs de pluie. Je grimpais dans ma petite chambre... Il y avait un bec de gaz que 
le vent faisait vaciller. Sa lueur faisait d'étranges soleils sur mes vitres.

Alors je me couchais. J'ouvrais un vieux livre plein d'histoires de diligences attaquées, de voyageurs perdus dans un chemin creux, d'histoires de veillées (La fontaine sans fond, la fée des grèves) ou bien un ancien recueil d'ornithologie dont les caractères écrasés se troublaient à la bougie ${ }^{28}$.

Si Bernard Baillaud souligne que Paulhan sème, dans ces trois nuits, des données de nature biographique (sa relation amoureuse avec Germaine Pascale, son désir de reprendre sa relation avec sa femme, Sala), il semble aussi que cette rêverie et le processus de remémoration qui l'accompagne puisent à une bibliothèque mentale où Meaulnes figure en belle place: aucune trace d'intertextualité évidente, mais des échos diffus, des lambeaux de textes, eux-mêmes modelés et informés par les souvenirs de la guerre, et l'amitié avec Uriet. Celui-ci, alors qu'il s'emploie à illustrer, en 1917, Le pont traversé, entend d'ailleurs aussi mettre au jour cette dialectique de la mémoire et de l'oubli. Comme Le Grand Meaulnes, sorte de clairobscur de la mémoire, dont le souvenir se perd en grande partie comme englouti par la monotonie des brumes de Sologne d'où se détacherait avec d'autant plus d'éclat, îlot de mémoire, la fête enchantée du domaine mystérieux ${ }^{29}$, Uriet entend, pour illustrer ce récit, tenir les images dans une tonalité «neutre et vague», où les figures et les choses évoquées, seules, seraient fortement éclairées. Comme Meaulnes et François Seurel, les deux amis caressent un rêve auquel la guerre de 1914 prête à présent une dimension tragique: écrire ce que François, comme Albert Uriet, désigne comme «notre roman d'aventure ${ }^{30}$.»

28. Lettre d'Uriet à Paulhan du $1^{\text {er }}$ septembre 1915, inédit, IMEC.

29. Point sur lequel a insisté Tiphaine Samoyault dans sa présentation du Grand Meaulnes (ouvr. cité, p. IX-XxxiI).

30. Voir la lettre d'Uriet à Paulhan d'octobre 1916 (inédit, IMEC) : «Je suis allé retrouver les deux Germaines - Elles avaient dormi tout le matin et étaient complètement remises — Nous avons causé de toi — Germaine P semblait tellement heureuse! Germaine H m'a dit — Ah! sûrement nous avons dépassé le Grand Meaulnes! (Quelle chose étonnante que nous ayons trouvé deux femmes aussi enthousiastes de notre grande aventure?)» 